\title{
Lung and kidney perfusion deficits diagnosed by dual-energy computed tomography in patients with COVID-19-related systemic microangiopathy
}

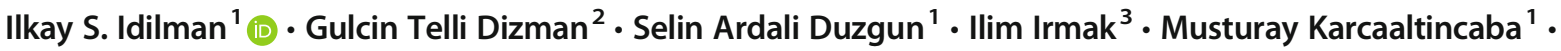 \\ Ahmet Cagkan Inkaya ${ }^{2}$. Figen Demirkazik ${ }^{1} \cdot$ Gamze Durhan $^{1} \cdot$ Meltem Gulsun Akpinar ${ }^{1} \cdot$ Orhan Macit Ariyurek $^{1}$. \\ Erhan Akpinar ${ }^{1} \cdot$ Jordi Rello $^{4} \cdot$ Murat Akova $^{2} \cdot$ Deniz Akata $^{1}$
}

Received: 22 May 2020 / Revised: 27 July 2020 / Accepted: 5 August 2020 / Published online: 29 August 2020

(C) European Society of Radiology 2020

\begin{abstract}
Objectives There is increasing evidence that thrombotic events occur in patients with coronavirus disease (COVID-19). We evaluated lung and kidney perfusion abnormalities in patients with COVID-19 by dual-energy computed tomography (DECT) and investigated the role of perfusion abnormalities on disease severity as a sign of microvascular obstruction.

Methods Thirty-one patients with COVID-19 who underwent pulmonary DECT angiography and were suspected of having pulmonary thromboembolism were included. Pulmonary and kidney images were reviewed. Patient characteristics and laboratory findings were compared between those with and without lung perfusion deficits (PDs).

Results DECT images showed PDs in eight patients (25.8\%), which were not overlapping with areas of ground-glass opacity or consolidation. Among these patients, two had pulmonary thromboembolism confirmed by CT angiography. Patients with PDs had a longer hospital stay $(p=0.14)$, higher intensive care unit admission rates $(p=0.02)$, and more severe disease $(p=0.01)$. In the PD group, serum ferritin, aspartate aminotransferase, fibrinogen, D-dimer, C-reactive protein, and troponin levels were significantly higher, whereas albumin level was lower $(p<0.05)$. D-dimer levels $\geq 0.485 \mu \mathrm{g} / \mathrm{L}$ predicted PD with $100 \%$ specificity and $87 \%$ sensitivity. Renal iodine maps showed heterogeneous enhancement consistent with perfusion abnormalities in 13 patients $(50 \%)$ with lower sodium levels $(p=0.03)$.

Conclusions We found that a large proportion of patients with mild-to-moderate COVID-19 had PDs in their lungs and kidneys, which may be suggestive of the presence of systemic microangiopathy with micro-thrombosis. These findings help in understanding the physiology of hypoxemia and may have implications in the management of patients with COVID-19, such as early indications of thromboprophylaxis or anticoagulants and optimizing oxygenation strategies.

Key Points

- Pulmonary perfusion abnormalities in COVID-19 patients, associated with disease severity, can be detected by pulmonary DECT.

- A cutoff value of $0.485 \mu \mathrm{g} / \mathrm{L}$ for D-dimer plasma levels predicted lung perfusion deficits with $100 \%$ specificity and $87 \%$ sensitivity (AUROC, 0.957).

- Perfusion abnormalities in the kidney are suggestive of a subclinical systemic microvascular obstruction in these patients.
\end{abstract}

Electronic supplementary material The online version of this article (https://doi.org/10.1007/s00330-020-07155-3) contains supplementary material, which is available to authorized users.

Murat Akova

akova.murat@gmail.com

Deniz Akata

akataden@gmail.com

1 Faculty of Medicine, Department of Radiology, Hacettepe University, Sihhiye, Ankara, Turkey
2 Faculty of Medicine, Department of Infectious Diseases and Clinical Microbiology, Hacettepe University, Sihhiye, Ankara, Turkey

3 Faculty of Medicine, Department of Chest Diseases, Hacettepe University, Ankara, Turkey

4 Department of General Area Intensive Medicine, University Hospital Vall d'Hebron, Barcelona, Spain 
Keywords COVID-19 · Computed tomography angiography · Lung · Pulmonary embolism

$\begin{array}{ll}\text { Abbreviations } \\ \text { COVID-19 } & \text { Coronavirus disease 2019 } \\ \text { CT } & \text { Computed tomography } \\ \text { DECT } & \text { Dual-energy computed tomography } \\ \text { GGO } & \text { Ground-glass opacity } \\ \text { HU } & \text { Hounsfield unit } \\ \text { ICU } & \text { Intensive care unit } \\ \text { LV } & \text { Left ventricle } \\ \text { PD } & \text { Perfusion deficit } \\ \text { PE } & \text { Pulmonary embolism } \\ \text { RV } & \text { Right ventricle } \\ \text { VNC } & \text { Virtual non-contrast }\end{array}$

\section{Introduction}

Radiological imaging of the lungs has become a diagnostic tool for the coronavirus disease (COVID-19) due to the low sensitivity of real-time reverse-transcription polymerase chain reaction (RT-PCR) tests from swab samples [1-3]. Several imaging characteristics have been described, including single or multiple focal ground-glass opacities (GGOs) in the lungs [4, 5]. Recently, multiorgan endotheliitis was identified in patients with COVID-19, which may be due to the widespread distribution and co-expression of ACE-2 and S-protein-specific proteases [6-8]. Microangiopathic lesions with thrombosis have been shown in pathological specimens from the lungs of critically ill patients with COVID-19 [9], and anticoagulant therapy has been shown to improve their prognosis [10]. However, these findings are based on recent pathological studies and it is evident that noninvasive diagnosis of the microvascular obstruction would be beneficial in the management of COVID-19 patients using early thromboprophylaxis.

Dual-energy computed tomography (DECT) allows the quantification of iodine uptake, which is a good surrogate for pulmonary parenchymal perfusion $[11,12]$. This technique can be used for CT angiography and it allows for the evaluation of pulmonary perfusion and the presence of pulmonary artery thrombus simultaneously, without increasing the radiation dose as compared with standard CT. Iodine maps obtained with this method can also be used to evaluate kidney perfusion, which are included at the caudal aspect of the DECT angiography. Here, we aimed to evaluate lung and kidney perfusion abnormalities in COVID-19 patients by dual-energy computed tomography (DECT) and to investigate the role of perfusion abnormalities on disease severity as a sign of microvascular obstruction.

\section{Materials and methods}

\section{Patients and clinical follow-up}

Thirty-one adults ( $>18$ years) diagnosed with COVID-19 and hospitalized between March 15, 2020, and April 20, 2020, in Hacettepe University Adult Hospital (a 1000-bed tertiary care facility), who underwent DECT angiography due to suspected pulmonary thromboembolism (PE) were analyzed. COVID19 was diagnosed using reverse-transcription polymerase chain reaction (RT-PCR) on nasopharyngeal smears. According to national guidelines, all RT-PCR-confirmed COVID-19 patients were considered for hospital admission. Electronic records of the Hacettepe University Hospital and follow-up charts of the patients were reviewed by the authors (II, GTD, ACI) and saved in an electronic database. Confirmed COVID-19 patients who were evaluated as needing treatment as per the national guidelines were admitted into isolation wards. A suspected PE was based on clinical findings and/or elevated D-dimer serum levels (>1000 ng/mL). Patients were informed about the radiological procedure and provided their informed consent. Pregnant women and those who did not give their consent were excluded. Clinical disease severity for COVID-19 was defined as proposed by Feng et al [13]. Briefly, patients are categorized into four types: type 1 had mild symptoms and no abnormal radiological findings, type 2 had moderate symptoms and evidence of pneumonia on chest $\mathrm{CT}$, type 3 patients had either a high respiratory rate ( $\geq 30 / \mathrm{min}$ ) or $\mathrm{SaO}_{2}(\leq 93 \%)$ or low oxygen partial pressure/ inspired oxygen fraction $(\leq 300 \mathrm{mmHg})$ in arterial blood, and type 4 patients needed mechanical ventilation and had shock or organ dysfunction needing intensive care unit (ICU) admission.

\section{CT acquisition protocol and DECT post-processing and image reconstruction}

The DECT angiography images were obtained by thirdgeneration dual-source CT (Somatom Force, Siemens Healthineers). Patients received 50-60 mL iohexol (Omnipaque 350; GE Healthcare) intravenously, at a rate of $4.0 \mathrm{~mL} / \mathrm{s}$ via an antecubital intravenous catheter, followed by a 40-mL saline chaser bolus. A region of interest (ROI) was placed over the pulmonary artery, and the acquisition was started when the ROI reached $100 \mathrm{HU}$ with a delay of $5 \mathrm{~s}$. The craniocaudal acquisition was set with the following parameters: 80/140 Sn kVp, modulated mA (CareDose 4D, Siemens Healthineers) with reference $80 \mathrm{mAs}$, rotation time $0.25 \mathrm{~s}$, with a pitch of 0.7 , and a collimation of $(64 \times 0.6) \mathrm{mm} \times 2$. 
Perfused blood volume (PBV) images and iodine maps were generated using DECT post-processing software ("Lung PBV" and "Virtual unenhanced" in syngo Dual Energy; Siemens Healthineers) on a dedicated workstation.

The DECT scanner generates three different series of images: $80-\mathrm{kV}$ images, $140-\mathrm{kV}$ images, and weighted-average images (similar to $120-\mathrm{kVp}$ scan of the abdomen). Images were loaded to a dedicated DE post-processing workstation (Syngo Via VB10; Siemens Medical Solutions). Using the Lung PBV application, iodine uptake distribution can be mapped to visualize perfusion. This calculation is based on the so-called three-material decomposition: Assuming that every voxel in the lung is composed of air, soft tissue, and iodine, the algorithm generates a map that encodes the iodine distribution in each individual CT voxel. To generate the lung perfusion maps, we placed an ROI on the pulmonary artery. We used a scale factor of 0.15 to normalize the perfusion of the lung parenchyma. In addition to lung PBV images, virtual non-contrast (VNC) image and iodine maps were obtained using the "virtual unenhanced" software. Iodine map can be superimposed on weighted-average or VNC images for the visualization of iodine uptake distribution and anatomic information simultaneously.

Morphologic images, lung perfusion maps, and iodine maps were analyzed by three experienced readers (with 14, 24, and 32 years of $\mathrm{CT}$ experience). The image quality of the perfusion map was recorded as either excellent (no artifacts), good (minor artifacts), moderate (still able to assess iodine distribution), or poor (impossible to assess iodine distribution). The pulmonary DECT angiography image quality was excellent in 2 , good in 21 , and moderate in 8 patients. The perfusion map images were then reviewed for the presence of any deficit. Perfusion map deficits were characterized as either overlapping with GGO or consolidation, not overlapping with GGO or consolidation, or band-like deficits consistent with artifacts, often due to cardiac motion or beam hardening from the contrast material within the superior vena cava or innominate vein. Lesions on the CT images above $1 \mathrm{~cm}$ were also evaluated and recorded as GGO or consolidation, and the iodine uptake of these lesions was measured with three elliptic rounds of interest (ROI) on the iodine map images and the mean value was calculated.

Lung CT images were classified according to the extent of GGOs and the presence of consolidation and crazy paving pattern in the lobes. CT scores were defined as follows: 0 (none), 1 (affecting less than $5 \%$ of the lobe), 2 (affecting 5-25\% of the lobe), 3 (affecting 26-49\% of the lobe), 4 (affecting 50-75\% of the lobe), and 5 (affecting $>75 \%$ of the lobe) [14]. If the crazy paving pattern or consolidation appeared in one lobe, the CT score was increased by 1 for each of them. Therefore, a maximum CT score of 7 was possible for each lobe. The total CT score was calculated by summing the five lobe scores (range from 0 to 35). Perfusion images were graded according to the extent of perfusion deficits (PDs). The grades were defined as follows: 0 (no PD), 1 (affecting only one area), 2 (affecting 1-3 PD areas), 3 (multiple bilateral PDs $\geq 4-10$ areas), and 4 (bilateral PDs disseminated in all segments covering $>50 \%$ of the total lung perfusion areas). Right ventricle (RV) and left ventricle (LV) diameters were measured from the axial slices showing the maximal distance between the intraventricular septum and endocardium. Following the measurements, the transverse $\mathrm{RV} / \mathrm{LV}$ ratio was calculated.

In 5 patients, pulmonary CTA examinations did not include more than $75 \%$ of the longitudinal diameter of the kidneys and were excluded from the kidney analyses. The kidneys were analyzed on VNC, iodine map, and mixed DECT images. An ROI was placed over the abdominal aorta to normalize the contrast enhancement before the analysis. Visual analyses of these images were performed by three radiologists in consensus. Homogeneous pattern on the iodine map is defined as a smooth appearance without low iodine uptake areas; heterogeneous pattern is defined as a mottled appearance with alternating low and high iodine uptake. Density measurements were made on the cortex of the kidney through the iodine map and VNC images. A freehand ROI was placed on the cortex of the kidney and density measurements were recorded. Mottled areas and adjacent normally enhancing parenchyma were also evaluated from the lower, mid, and upper poles of the kidney in the diffuse heterogeneously enhancing kidneys and from the low perfused areas in the focal heterogeneously enhancing kidneys by using a circular region-of-interest of approximately $5 \mathrm{~mm}^{2}$ and the average was calculated for each.

\section{Statistical analysis}

Categorical data were presented as numbers (percentages) and continuous variables are expressed as means \pm standard deviations unless otherwise stated. Categorical data were compared using the Pearson chi-square test/Fisher's exact test and continuous variables were compared using Student's $t$ test or Mann-Whitney $U$ test according to the distribution of data. The degree of association between continuous and/or ordinal variables was calculated using Pearson's correlation coefficient or Spearman's rho analysis according to the distribution of data. A one-way analysis of variance (ANOVA) or Kruskal-Wallis analysis was performed to compare continuous variables in different PD grades. A two-tailed $p$ value of $<0.05$ was considered statistically significant. A receiver operating characteristic (ROC) curve analysis was performed to define the value of laboratory parameters in predicting PD.

\section{Results}

A total of 31 patients (M/F 16/15) with COVID-19, who underwent pulmonary DECT angiography for a suspected $\mathrm{PE}$, were retrospectively analyzed. The mean age of the 
Table 1 Characteristics of patients with and without lung perfusion deficit

\begin{tabular}{|c|c|c|c|c|}
\hline & All patients & $\begin{array}{l}\text { Patients with lung perfusion } \\
\text { deficit, } n=8\end{array}$ & $\begin{array}{l}\text { Patients without perfusion } \\
\text { deficit, } n=23\end{array}$ & $p$ value \\
\hline Age (years) mean $\pm \mathrm{SD}$ & $39.2 \pm 12$ & $42.8 \pm 7.03$ & $38.0 \pm 13.59$ & $0.348 *$ \\
\hline \multicolumn{5}{|l|}{ Sex } \\
\hline Male, $n(\%)$ & $17(54.8)$ & $4(50)$ & $13(56.5)$ & $0.750 * * *$ \\
\hline \multicolumn{5}{|l|}{ No of comorbidities, $n(\%)$} \\
\hline $\begin{array}{l}<2 \\
\geq 2\end{array}$ & $\begin{array}{l}8(25.8) \\
1(3.2)\end{array}$ & $\begin{array}{l}3(37.5) \\
0\end{array}$ & $\begin{array}{l}5(21.7) \\
1(4.3)\end{array}$ & $0.453 * * *$ \\
\hline Obesity, $n(\%)$ & $5(16.1)$ & $3(37.5)$ & $2(8.7)$ & $0.056 * * *$ \\
\hline $\mathrm{BMI}\left(\mathrm{kg} / \mathrm{m}^{2}\right)$ mean $\pm \mathrm{SD}$ & $26.69 \pm 3.49$ & $28.85 \pm 3.77$ & $25.94 \pm 3.12$ & $0.040^{*}$ \\
\hline Active smokers, $n(\%)$ & $8(25.8)$ & $2(25)$ & $6(26)$ & $0.952 * * *$ \\
\hline ACE inhibitors or ARB, $n(\%)$ & $1(3.2)$ & 0 & $1(4.3)$ & $0.549 * * *$ \\
\hline \multicolumn{5}{|l|}{ Clinical disease severity, $n(\%)$} \\
\hline Type 1 & $8(25.8)$ & $1(12.5)$ & $7(30.4)$ & $0.010 * * *$ \\
\hline Type 2 & $18(58.1)$ & $3(37.5)$ & $15(65.2)$ & $0.010 * * *$ \\
\hline Type 3 & $5(16.1)$ & $4(50)$ & $1(4.3)$ & $0.010 * * *$ \\
\hline $\mathrm{CT}$ score mean $\pm \mathrm{SD}$ & $7.6 \pm 7.1$ & $13.3 \pm 8.2$ & $5 \pm 5.4$ & $0.025 *$ \\
\hline $\mathrm{RV} / \mathrm{LV}$ ratio mean $\pm \mathrm{SD}$ & $0.86 \pm 0.09$ & $0.92 \pm 0.11$ & $0.84 \pm 0.08$ & $0.030^{*}$ \\
\hline \multicolumn{5}{|l|}{ Symptom at presentation, $n(\%)$} \\
\hline Asymptomatic & $5(16.1)$ & $7(87.5)$ & $19(82.6)$ & $0.746 * * *$ \\
\hline Dry cough & $20(64.5)$ & $6(75)$ & $14(60.8)$ & $0.472 * * *$ \\
\hline Weakness & $17(54.8)$ & $5(62.5)$ & $12(52.1)$ & $0.613 * * *$ \\
\hline Myalgia & $12(38.7)$ & $4(50)$ & $8(34.7)$ & $0.447 * * *$ \\
\hline Fever & $10(32.3)$ & $3(37.5)$ & $7(30.4)$ & $0.713 * * *$ \\
\hline Sore throat & $7(22.6)$ & $4(50)$ & $3(13)$ & $0.031 * * *$ \\
\hline Dyspnea & $5(16.1)$ & $3(37.5)$ & $2(8.6)$ & $0.056 * * *$ \\
\hline Diarrhea & $5(16.1)$ & $1(12.5)$ & $4(17.3)$ & $0.746 * * *$ \\
\hline Headache & $5(16.1)$ & $1(12.5)$ & $4(17.3)$ & $0.746 * * *$ \\
\hline Nasal discharge & $3(9.7)$ & 0 & $3(13)$ & $0.282 * * *$ \\
\hline Chest pain & $3(9.7)$ & 0 & $3(13)$ & $0.282 * * *$ \\
\hline Hemoptysis & $1(3.2)$ & $1(12.5)$ & 0 & $0.085 * * *$ \\
\hline Fever at admission $\left({ }^{\circ} \mathrm{C}\right)$ mean $\pm \mathrm{SD}$ & $37 \pm 0.7$ & $37.4 \pm 0.8$ & $37 \pm 0.4$ & $1 * *$ \\
\hline Respiratory rate/min at admission mean $\pm \mathrm{SD}$ & $19.7 \pm 4.1$ & $21.5 \pm 4.87$ & $19.17 \pm 3.84$ & $0.248 * *$ \\
\hline Length of hospital stay (days) mean $\pm \mathrm{SD}$ & $8.6 \pm 6.8$ & $12.25 \pm 8.81$ & $6.83 \pm 5.04$ & $0.138 * *$ \\
\hline Length of ICU stay (days) mean $\pm \mathrm{SD}$ & $5.2 \pm 3.8$ & $6.67 \pm 3.21$ & 1 & $0.266 * *$ \\
\hline ICU admission, $n(\%)$ & $4(12.3 \%)$ & $3(37.5 \%)$ & $1(4.3 \%)$ & $0.043 * * *$ \\
\hline Symptom duration before DECT (days, median, IQR) & $3(2-11)$ & $11.5(4-18)$ & $3(1.5-5)$ & $0.034 * *$ \\
\hline DECT within 5 days after symptom onset, $n(\%)$ & $21(67.7)$ & $3(37.5)$ & $18(85.7)$ & $0.034 * * *$ \\
\hline Oxygen supplementation (L/min) (median, IQR) & $3(2-5)$ & $3(2-5)$ & $3.5(2-5)$ & $0.800 * *$ \\
\hline Prone position, $n(\%)$ & $4(12.9)$ & $3(37.5)$ & $1(4.3)$ & $0.016^{* * *}$ \\
\hline Oxygen therapy, $n(\%)$ & $6(19.3)$ & $4(50)$ & $2(8.6)$ & $0.011 * * *$ \\
\hline \multicolumn{5}{|l|}{ Antiviral therapy, $n(\%)$} \\
\hline No drug & $4(12.9)$ & 0 & $4(17.3)$ & $0.209 * * *$ \\
\hline Hydroxychloroquine + azithromycin & $18(58.1)$ & $5(62.5)$ & $13(56.5)$ & $0.209 * * *$ \\
\hline Hydroxychloroquine + azithromycin + favipiravir & $5(16.1)$ & $2(25)$ & $3(13)$ & $0.209 * * *$ \\
\hline Hydroxychloroquine & $3(9.7)$ & 0 & $3(13)$ & $0.209 * * *$ \\
\hline $\begin{array}{l}\text { Hydroxychloroquine }+ \text { azithromycin }+ \text { favipiravir }+ \\
\text { lopinavir/ritonavir }\end{array}$ & $1(3.2)$ & $1(12.5)$ & 0 & $0.209 * * *$ \\
\hline
\end{tabular}

Italics represent statistically significant results

Mean $\pm \mathrm{SD}$, median (IQR) were given. *Student's $t$ test, **Mann-Whitney $U$ test, ***Pearson's chi-square/Fisher's exact test were used 


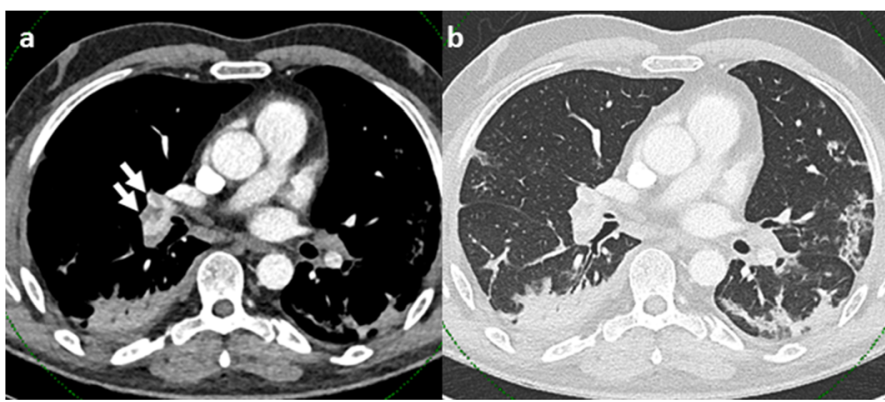

Fig. 1 A 47-year-old male with coronavirus disease 2019 (COVID 19). Pulmonary thromboembolism (PE) can be seen in the right lobar and segmental pulmonary arteries (arrows) on pulmonary dual-energy computed tomography (DECT) angiography images (a and b). On the perfusion map images (c), perfusion deficits due to the PE (arrows) can be

participants was $39.2 \pm 12$ years (range 23-65 years) (Table 1). All CT scans were performed in non-intubated patients, and the median number of days from the first symptom onset to $\mathrm{CT}$ scan detection was 3 days (IQR 2 to 11 ). In the analysis of the lung CT images, seven patients had no lung lesions with no PDs, and the mean CT score was 7.6 $\pm 7.1(0$ $22)$. There were significantly positive correlations between the CT score and age $(p=0.01, r=0.468)$ and severity of the disease $(p<0.001, r=0.760$, Supplementary Table 1$)$.

DECT angiography identified overt PE in only two patients (6.5\%). In the lung perfusion maps, these patients had PDs corresponding to the areas with vascular occlusion and areas of decreased perfusion without visible thrombus (Fig. 1). Apart from these two cases, PDs were visualized in six more patients who had no detectable emboli in the pulmonary arteries (Fig. 2). In total, there were 8 patients with PDs and 2 patients among them had gross filling defects in the pulmonary arteries. The PDs did not overlap with GGOs or consolidation. In the subgroup analysis, patients with PDs had prolonged hospital stays (12.28 \pm 8.81 vs $6.83 \pm 5.04$ days, $p=0.138)$, higher rates of ICU ad-

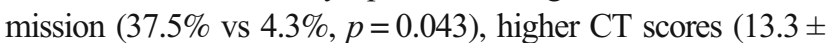
8.2 vs $5 \pm 5.4, p=0.025$ ), and a more severe (type 3 ) disease $(50 \%$ vs $4.3 \%, p=0.010)$. The only significantly frequent clinical symptom in the patients with PDs was sore throat $(50 \%$ versus $13 \%, p=0.031)$. BMI was higher in patients with PDs $\left(28.85 \pm 3.77\right.$ vs $\left.25.94 \pm 3.12 \mathrm{~kg} / \mathrm{m}^{2}, p=0.040\right)$. Smoking status was similar in the two groups $(25 \%$ vs $26 \%, p=0.952)$ (Table 1$)$.

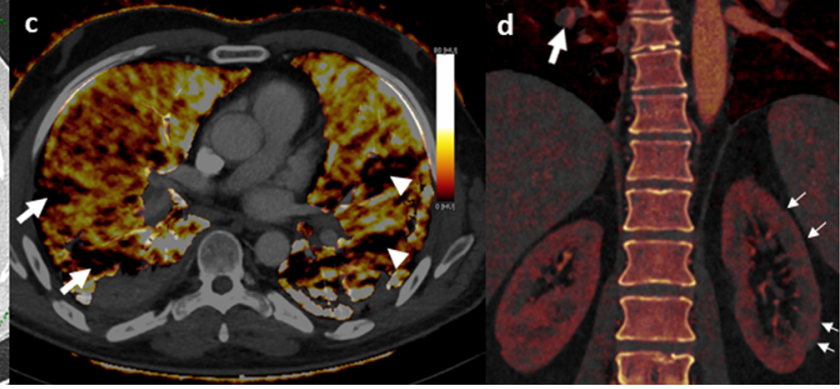

seen in the right lung. There are also perfusion deficits in the left lobe, which are not associated with vessel occlusion or parenchymal findings (arrowheads). On coronal renal perfusion images (d), heterogeneous kidney enhancement (arrows), and a PE in the right lower lobe pulmonary artery (large arrow) can be seen

Ferritin, AST, fibrinogen, D-dimer, CRP, and troponin plasma levels were significantly higher, and albumin levels were significantly lower in patients with PDs (Table 2). A receiver operator characteristic $(\mathrm{ROC})$ curve analysis revealed that D-dimer plasma levels $\geq 0.485 \mu \mathrm{g} / \mathrm{L}$ predicted lung PD with $100 \%$ specificity and $87 \%$ sensitivity (area under the (AU)ROC, 0.957) and with a positive predictive value of $80 \%$ and a negative predictive value of $100 \%$ (Fig. 3). When we grouped patients according to the extent of PDs, there were statistically significant differences in terms of clinical classification, CT score, presenting symptoms like sore throat, and the need for oxygen therapy (Table 3). Ddimer was the only laboratory variable related to $\operatorname{PD}(p=0.01)$.

Among 80 lung lesions, 38 lesions were GGOs, and the remaining 42 lesions were areas of consolidation. The average VNC density and iodine uptake of these lesions were $820.2 \mathrm{HU} \pm 40.9 \mathrm{HU}$ and $22.3 \mathrm{HU} \pm 12.4 \mathrm{HU}$, respectively. The average VNC of GGOs was $-400.9 \pm 155.7 \mathrm{HU}$ and consolidation was $-114.2 \pm 111.4 \mathrm{HU}$. Iodine uptake was significantly increased in consolidations compared with GGOs, showing more contrast enhancement in consolidations $(45.1 \pm 21.2 \mathrm{HU}$ vs $29.3 \pm 13.9 \mathrm{HU}, p=0.002$, Fig. 4$)$.

All the patients survived through the study period and were discharged from the hospital. We repeated the DECT imaging in two patients within 1 month. Previous PDs were improved in the first patient (Fig. 5). PDs in the second patient who had a pulmonary thromboembolism were improved with stable findings related to the emboli.
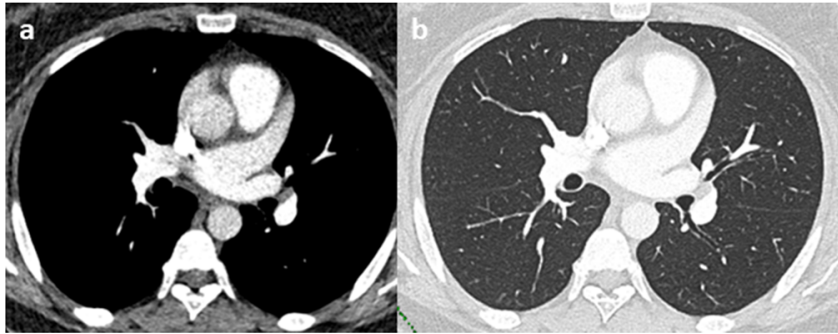

Fig. 2 A 36-year-old female with coronavirus disease 2019 (COVID 19). No evidence of a PE can be seen on contrast-enhanced dual-energy computed tomography (DECT) angiography (a and b). On the

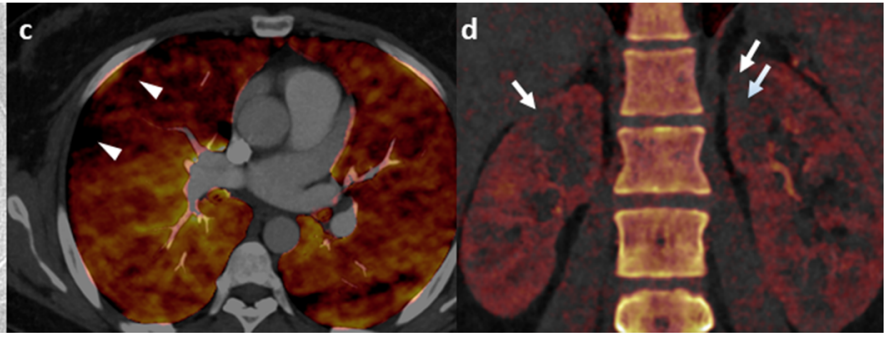

perfusion map images (c), perfusion deficits (arrowheads) can be seen in both lungs. On the coronal renal perfusion images (d), heterogeneous kidney enhancement with perfusion deficits (arrows) can be seen 
Table 2 Laboratory findings in patients with and without lung perfusion deficit

\begin{tabular}{|c|c|c|c|}
\hline & $\begin{array}{l}\text { Patients with } \\
\text { perfusion deficit, } n=8\end{array}$ & $\begin{array}{l}\text { Patients without } \\
\text { perfusion deficit, } n=23\end{array}$ & $p$ value \\
\hline Leukocyte count $\left(\times 10^{9} / \mathrm{L}\right)$ & $5.2 \pm 1.1$ & $6 \pm 3.3$ & $0.982 * *$ \\
\hline Neutrophil count $\left(\times 10^{9} / \mathrm{L}\right)$ & $\begin{array}{l}5.6(4-6) \\
3.6 \pm 1.2\end{array}$ & $\begin{array}{l}5.3(3.6-7.2) \\
3.7 \pm 2.8\end{array}$ & $0.411 * *$ \\
\hline \multirow[t]{2}{*}{ Lymphocyte count $\left(\times 10^{9} / \mathrm{L}\right)$} & $\begin{array}{l}3.5(2.7-4.7) \\
1.1 \pm 3.7\end{array}$ & $\begin{array}{l}2.8(2.1-4.5) \\
1.6 \pm 7.2\end{array}$ & $0.063 *$ \\
\hline & $\begin{array}{l}1.2(0.8-1.3) \\
4.6 \pm 4.6\end{array}$ & $\begin{array}{l}1.5(1.1-2) \\
2.6 \pm 1.7\end{array}$ & $0.145 * *$ \\
\hline Neutrophil-to-lymphocyte ratio (NLR) & $\begin{array}{r}3(2.5-4.1) \\
185.8 \pm 80.7\end{array}$ & $\begin{aligned} 2.5(1.4-3.6) \\
204.6 \pm 46\end{aligned}$ & $0.074 * *$ \\
\hline Platelet count $\left(\times 10^{9} / \mathrm{L}\right)$ & $\begin{array}{l}169(151.5-180.5) \\
14.3 \pm 2.3\end{array}$ & $\begin{array}{c}202(170-230) \\
13.8 \pm 1.9\end{array}$ & $0.606^{*}$ \\
\hline Hemoglobin (g/dL) & $\begin{array}{l}13.9(12.8-16.5) \\
583.3 \pm 736.5\end{array}$ & $\begin{array}{c}14(12.8-15.2) \\
106.1 \pm 167.4\end{array}$ & $0.012 * *$ \\
\hline Ferritin $(\mu \mathrm{g} / \mathrm{L})$ & $\begin{array}{l}246.5(88.4-1095.8) \\
\quad 4.14 \pm 4.1\end{array}$ & $\begin{array}{l}50(30.3-97.8) \\
1 \pm 1.3\end{array}$ & $0.013 * *$ \\
\hline $\mathrm{CRP}(\mathrm{mg} / \mathrm{dL})$ & $\begin{array}{l}2.4(0.8-7.6) \\
0.05 \pm 0.02\end{array}$ & $\begin{array}{l}0.7(0.3-0.9) \\
0.04 \pm 0.06\end{array}$ & $0.084 * *$ \\
\hline Procalcitonin $(\mathrm{ng} / \mathrm{mL})$ & $\begin{array}{l}0.05(0.03-0.06) \\
40 \pm 19.7\end{array}$ & $\begin{array}{l}0.03(0.02-0.04) \\
25.4 \pm 8.3\end{array}$ & $0.039 * *$ \\
\hline AST (U/L) & $34.5(25.3-59.8)$ & $24(21-27)$ & \\
\hline \multirow[t]{2}{*}{$\operatorname{ALT}(\mathrm{U} / \mathrm{L})$} & $29.4 \pm 11.5$ & $23.9 \pm 15.1$ & $0.147 * *$ \\
\hline & $\begin{array}{c}26(24.3-38) \\
0.7 \pm 0.3\end{array}$ & $\begin{array}{c}19(14-32) \\
0.5 \pm 0.3\end{array}$ & $0.106^{* *}$ \\
\hline Total bilirubin (mg/dL) & $\begin{array}{l}0.6(0.5-0.9) \\
3.8 \pm 0.4\end{array}$ & $\begin{array}{l}0.5(0.4-0.6) \\
4.2 \pm 0.4\end{array}$ & $0.046^{*}$ \\
\hline Albumin $(\mathrm{g} / \mathrm{dL})$ & $3.9(3.4-4.2)$ & $4.2(4-4.4)$ & \\
\hline \multirow[t]{2}{*}{ LDH (U/L) } & $333.3 \pm 272.6$ & $180.4 \pm 37.9$ & $0.055^{* *}$ \\
\hline & $\begin{array}{l}241.5(25.3-59.8) \\
2.1 \pm 2.8\end{array}$ & $\begin{array}{l}171.5(154-202.5) \\
0.3 \pm 0.2\end{array}$ & $<0.001 * *$ \\
\hline D-dimer $(\mu \mathrm{g} / \mathrm{L})$ & $\begin{array}{r}1.1(0.6-1.9) \\
447.6 \pm 139.6\end{array}$ & $\begin{aligned} & 0.3(0.2-0.4) \\
& 337.4 \pm 79.2\end{aligned}$ & $0.013 *$ \\
\hline Fibrinogen (mg/dL) & $\begin{array}{l}416(338.3-596) \\
465.3 \pm 579\end{array}$ & $\begin{array}{l}312(269-430) \\
126.9 \pm 87.5\end{array}$ & $0.281 * *$ \\
\hline \multirow[t]{2}{*}{ Blood urea nitrogen (mg/dL) } & $\begin{array}{l}171.5(65.5-876.3) \\
10.9 \pm 3.9\end{array}$ & $\begin{array}{l}102(63.8-179.8) \\
12.4 \pm 4.2\end{array}$ & $0.408 *$ \\
\hline & $11.7(8-12.7)$ & $12.3(9.5-15.3)$ & \\
\hline \multirow[t]{2}{*}{ Creatinine $(\mathrm{mg} / \mathrm{dL})$} & $0.7 \pm 0.2$ & $0.7 \pm 0.1$ & $0.779 *$ \\
\hline & $\begin{aligned} & 0.7(0.6-0.8) \\
& 136.9 \pm 3.1\end{aligned}$ & $\begin{aligned} 0.7 & (0.7-0.8) \\
138.2 & \pm 2.4\end{aligned}$ & $0.254 *$ \\
\hline Sodium (mEq/L) & $136.5(135-139)$ & $139(138-140)$ & \\
\hline \multirow{2}{*}{ Potassium (mEq/L) } & $3.9 \pm 0.3$ & $4.1 \pm 0.4$ & $0.354 * *$ \\
\hline & $3.8(3.8-4.2)$ & $4(3.8-4.3)$ & \\
\hline \multirow[t]{2}{*}{ Troponin (ng/L) } & $4.4 \pm 2.4$ & $3 \pm 1.5$ & $0.039 * *$ \\
\hline & $3.5(2.6-6)$ & $2.3(2.3-3.6)$ & \\
\hline
\end{tabular}

Italics represent statistically significant results

Mean \pm standard deviation, median (IQR) were given

*Student's $\mathrm{t}$ test or **Mann-Whitney $U$ tests were used
A total of 51 kidneys were evaluated in 26 patients (one patient had a single kidney). In 13 patients (50\%), there was a heterogeneous enhancement in the cortex of the kidneys. Among them, focal heterogeneous enhancement in the left upper pole of the kidney was detected in two patients and a diffuse heterogeneous enhancement in the cortex of both kidneys was detected in 11 patients. The typical findings were sub-centimeter moth-eaten like low iodine uptake areas in the cortex of the kidneys. None of the patients had evidence of abnormal kidney function. 


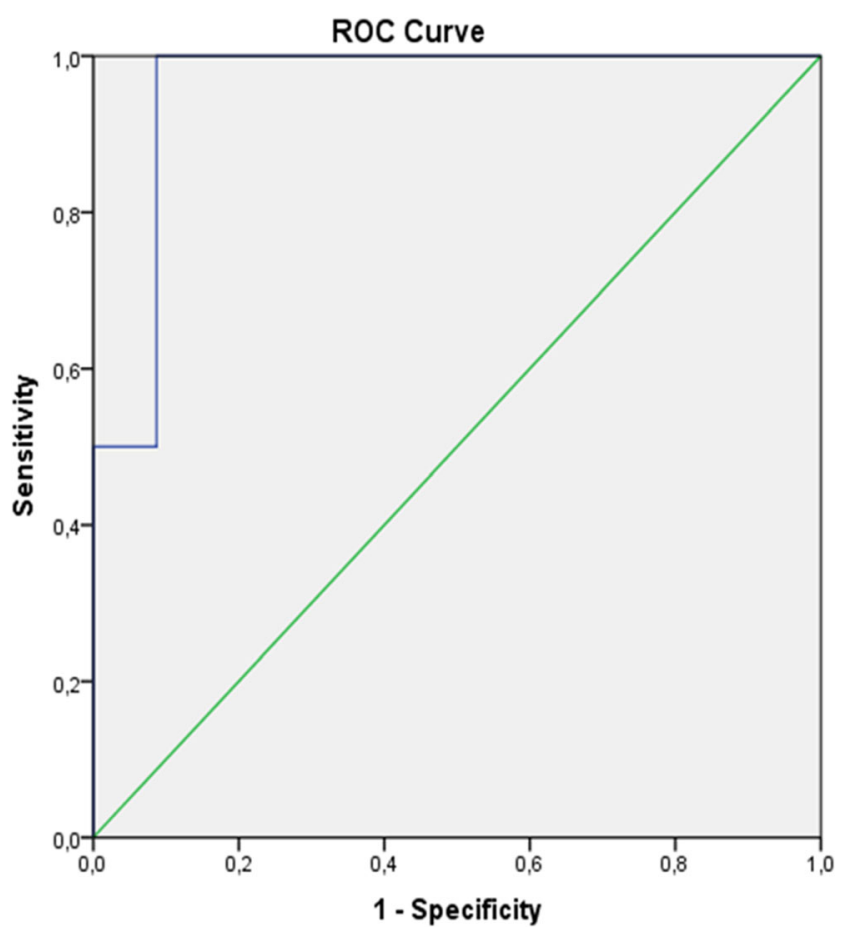

Fig. 3 ROC curve of D-dimer for differentiation of patients with PDs

Serum sodium levels were lower $(p=0.03)$ in these patients compared with those who had normal kidney perfusions (Supplementary Table 2). The overall iodine uptake of the heterogeneous kidneys $(n=24)$ was lower in comparison with the normal ones $(n=27)(73.9 \mathrm{HU}$

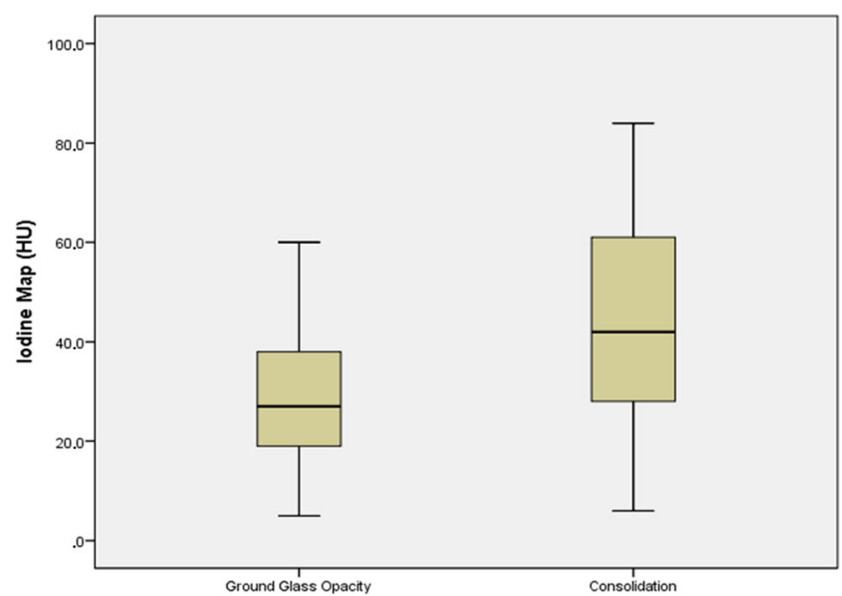

Fig. 4 Boxplot shows the difference between iodine uptake of GGOs and consolidations

$\pm 26 \mathrm{HU}$ vs $133.7 \mathrm{HU} \pm 28.3 \mathrm{HU}, p<0.001)$. Quantitative measurements revealed a significantly lower perfusion in the mottled areas of patients with a heterogeneous pattern $(25.4 \mathrm{HU} \pm 12.6 \mathrm{HU}$ vs $110.7 \mathrm{HU} \pm 31 \mathrm{HU}$, respectively, $p<0.001)$. However, these low perfused areas had significantly higher VNC values compared with the normal parenchyma (52 $\mathrm{HU} \pm 19 \mathrm{HU}$ vs $14.3 \mathrm{HU} \pm$ $9.3 \mathrm{HU}, p<0.001)$. The presence of heterogeneous kidney enhancement in patients with pulmonary PDs was slightly higher $(3 / 5,60 \%)$ in comparison with that in patients without pulmonary PDs $(10 / 21,47.6 \%)$ with no statistically significant difference $(p=1)$.

Table 3 Characteristics of patients according to the perfusion deficit grade

\begin{tabular}{|c|c|c|c|c|c|c|}
\hline & $\mathrm{PD}$ grade $0, n=23$ & PD grade $1, n=1$ & PD grade $2, n=1$ & PD grade $3, n=3$ & $\mathrm{PD}$ grade $4, n=3$ & $p$ value \\
\hline \multicolumn{7}{|l|}{ Clinical classification, $n(\%)$} \\
\hline Type 1 & $7(30.4)$ & 1 & 0 & 0 & 0 & \\
\hline Type 2 & $15(65.2)$ & 0 & $1(100)$ & $1(33.3)$ & $1(33.3)$ & $0.024 * * *$ \\
\hline Type 3 & $1(4.3)$ & 0 & 0 & $2(66.7)$ & $2(66.7)$ & \\
\hline CT scoreII & $5 \pm 5.4$ & 2 & 6 & $17 \pm 5.6$ & $15.7 \pm 9.2$ & $0.005 *$ \\
\hline \multicolumn{7}{|l|}{ Symptom at presentation, $n(\%)$} \\
\hline Sore throat & $3(13)$ & 0 & 0 & $3(100)$ & $1(33.3)$ & $0.015 * * *$ \\
\hline $\begin{array}{l}\text { Day from symptom onset to } \\
\text { DECT, median (IQR) }\end{array}$ & $3(1-5)$ & 5 & 3 & $15(10-22)$ & $13(1-21)$ & $0.147 * *$ \\
\hline $\mathrm{CT} \leq 5$ days, $n(\%)$ & $18(78.3)$ & $1(100)$ & $1(100)$ & 0 & $1(33.3)$ & $0.040 * * *$ \\
\hline Oxygen supplementation, $n(\%)$ & $2(8.7)$ & 0 & 0 & $2(66.7)$ & $2(66.7)$ & $0.029 * * *$ \\
\hline Ferritin $(\mu \mathrm{g} / \mathrm{L})^{\mathbb{I}}$ & $106 \pm 167$ & 53.2 & 194 & $778 \pm 1151$ & $694 \pm 529$ & $0.088 * *$ \\
\hline $\mathrm{CRP}(\mathrm{mg} / \mathrm{dL})^{\mathrm{TI}}$ & $0.97 \pm 1.34$ & 0.5 & 2.57 & $4.87 \pm 5.87$ & $5.15 \pm 3.83$ & $0.081 * *$ \\
\hline Albumin $(\mathrm{g} / \mathrm{dL})^{\mathrm{TI}}$ & $4.15 \pm 0.37$ & 4 & 3.6 & $3.7 \pm 0.4$ & $3.8 \pm 0.6$ & $0.289^{*}$ \\
\hline D-dimer $(\mu \mathrm{g} / \mathrm{L})^{\mathrm{TI}}$ & $0.34 \pm 0.23$ & 0.74 & 1.69 & $3.38 \pm 4.72$ & $1.28 \pm 0.68$ & $0.006^{* *}$ \\
\hline Fibrinogen $(\mathrm{mg} / \mathrm{dL})^{\mathrm{TI}}$ & $337 \pm 79$ & 329 & 563 & $479 \pm 117$ & $416 \pm 194$ & $0.182 *$ \\
\hline Troponin $(\mathrm{ng} / \mathrm{L})^{\mathrm{II}}$ & $3.03 \pm 1.47$ & 2.5 & 3.6 & $4.76 \pm 3.93$ & $5 \pm 1.41$ & $0.203 * *$ \\
\hline
\end{tabular}

Italics represent statistically significant results

${ }^{\text {II }}$ Mean $\pm \mathrm{SD}$, one-way ANOVA *Kruskal-Wallis ** or Pearson's chi-square/Fisher's exact*** tests were used

$D E C T$, dual-energy computed tomography 
Fig. 5 A 47-year-old male with coronavirus disease 2019 (COVID-19). The dual-energy computed tomography (DECT) angiography perfusion map (a) demonstrates multiple perfusion deficits, especially in the right lung (arrowheads). These areas do not match with the multiple ground-glass opacities seen on conventional CT (b). Follow-up DECT angiography 25 days after the first exam. Perfusion map (c) and conventional CT images (d) demonstrate a more prominent regression in the perfusion deficits compared with the conventional CT findings

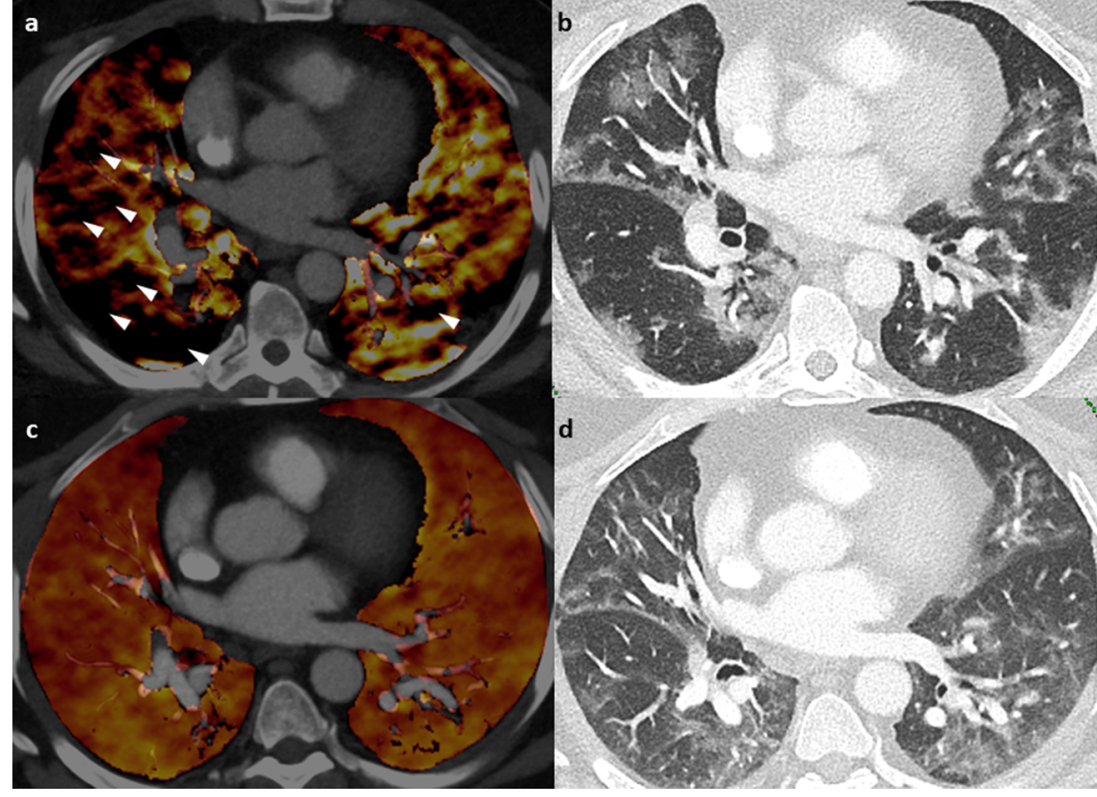

\section{Discussion}

Among 31 non-intubated patients with mild COVID-19, DECT angiography demonstrated PDs in the lungs of $25.8 \%$ of patients and in half of the kidneys assessed. Only two of them had major lung vascular occlusion leading to PE, and none had major renal dysfunction. In the quantitative analysis, we observed lower relative enhancement values in patients with PDs in comparison with patients having no PDs, confirming the decreased perfusion in this patient group. Although the clinical signs were not suggestive of the presence of perfusion abnormalities, they were significantly associated with some biomarkers such as higher D-dimer plasma concentrations, CRP, ferritin, or troponin. Increased D-dimer levels were demonstrated to be an early clinical predictor of perfusion abnormalities, with a threshold of $0.485 \mu \mathrm{g} / \mathrm{L}$ predicting PD with $100 \%$ specificity and $87 \%$ sensitivity. In the analysis of 80 lung lesions observed in these patients, we observed a lower iodine uptake in the GGOs compared with the consolidations, which is consistent with the increased perfusion in the lesions during the latter phase of the disease.

A recent study by Gervaise et al observed acute PE in 18\% of non-hospitalized COVID-19 patients [15]. In this study, no statistically significant difference was observed in terms of severity and radiological features of COVID-19 pneumonia between patients with or without acute PE. Another study reported that $23 \%$ of patients with COVID-19 who had severe clinical features suffered from a PE. These scholars suggested the use of contrast-enhanced CT rather than routine noncontrast $\mathrm{CT}$ in these patients [16]. We observed overt PE in only two patients $(6.5 \%)$ with mild clinical symptoms. However, we demonstrated PDs in $25.8 \%$ of our patient population, with worse clinical and laboratory parameters.
Iodine maps and perfusion blood volume (PBV) images generated by DECT provide information about perfusion of the lungs by assessing iodine distribution in the lungs. In PE, PBV images demonstrate acute infarction as a peripheral wedgeshaped area of non-enhancement that is usually bigger than the pulmonary opacity seen on conventional CT images [17]. In patients with pneumonia and atelectasis, the abnormalities seen on conventional CT images and PBV are similar in size, with heterogeneously decreased or increased iodine distribution in the former and increased iodine distribution in the latter [18].

In our study population, we demonstrated PDs in lungs that do not overlap with GGOs or consolidation and are also not associated with the occlusion of major vessels. A similar observation was recently described [17]. Angiopathic microvascular occlusion has been proposed as the underlying mechanism [19]. Another recent study showed fibrin thrombi of small arterial vessels less than $1 \mathrm{~mm}$ in diameter in 33 of 38 patients with high D-dimer levels [20]. Increased D-dimer levels and higher RV/LV ratio which is an indirect sign of $\mathrm{RV}$ overload in our patients with PDs are supportive of these observations. DECT can improve the detection of small pulmonary emboli that may otherwise be missed with conventional CT angiography [21]. In our population, we observed lower iodine uptake in GGOs compared with consolidations. This finding can be explained by the predominant effect of edema in the early phase of the disease and increased perfusion in the later phases.

Viral infections can trigger systemic inflammation and activate the coagulation cascade through different mechanisms [22]. Inflammatory cytokines, complement activation, endothelial infection, and endotheliitis are the leading causes of thrombosis in patients with COVID-19 [8, 23]. Furthermore, platelet activation through $\mathrm{Fc}$ receptor binding may also initiate a coagulation cascade [24]. 
Therefore, thromboprophylaxis with low molecular weight heparin (LMWH) has been proposed in these patients unless there are contraindications [25]. In this study, none of the patients received thromboprophylaxis before CT imaging. Subsequently, patients with a confirmed PE received anticoagulant treatment, and the remaining patients received thromboprophylaxis with LMWH. Early commencement of thromboprophylaxis might have also contributed to the favorable survival rate of our patients. In addition, hydroxychloroquine treatment used in most of the patients might have prevented the progression of the PDs to major thromboembolic events as this drug has been previously shown to prevent thrombosis in patients with systemic lupus erythematosus and antiphospholipid antibodies [26].

We also showed heterogeneous kidney enhancement in DECT analysis in 13 of $26(50 \%)$ patients, which is suggestive of microvascular occlusion. The patients had no detectable major kidney dysfunction, but lower serum sodium levels compared with the patients with normal kidneys. ACE2, the functional receptor of severe acute respiratory syndrome coronavirus 2 (SARS-CoV-2), and its S-protein priming proteases have been found to be widespread in the vascular endothelium and several organ systems [8]. Together with the findings of a recent study that showed the presence of viral elements within endothelial cells, a COVID-19 endotheliitis could explain the systemic impaired microcirculatory function in different vascular beds. In support of this proposition, another recent study observed tissue damage consistent with complement-mediated microvascular injury in the lungs and/ or skin of five individuals with severe COVID-19 [23]. Another study showed the direct parenchymal infection of tubular epithelial cells and podocytes with marked erythrocyte aggregation in patients with severe COVID-19 [27]. The observed high VNC values of the hypo-perfused areas may explain such aggregates or thrombosis in our study population. None of our patients developed kidney dysfunction which may be explained with the relatively small percentage of kidney involvement and the early LMWH treatment.

There are several limitations of this study. We did not perform a dedicated CT perfusion study on the lungs and kidneys. However, DECT is a widely used quantitative technique and enables the measurement of iodine uptake as a reliable indirect evidence of perfusion. In addition, the scanning protocol was not optimized for abdominal perfusion $\mathrm{CT}$, as scanning delay was set with a ROI placed on the pulmonary artery. However, we observed focal low perfused areas with increased statistically significant VNC values, which show that our findings are not related to technical factors. Secondly, our sample size was small, which can be explained by the fact that pulmonary CTA with DECT is not a routine CT protocol in patients with COVID-19 and the retrospective nature of the study.
The third limitation is that our sample contained patients with mild COVID-19 who did not require intubation and/ or mechanical ventilation. The patients in this cohort were relatively young with few comorbidities, and findings may differ under different conditions. On the other hand, this cohort strengthens the thesis of COVID-19-induced perfusion abnormalities. A selection bias cannot be completely ruled out. Similarly, the data cannot be generalized because cohorts with different symptoms or duration of illness can present other abnormalities. We observed a slightly higher rate of heterogeneous kidney enhancement in patients with pulmonary PDs $(60 \%)$ in comparison with patients without pulmonary PDs (47.6\%). This observation could provide strong imaging evidence supporting the presence of systemic microvascular angiopathy in COVID-19 along with RV enlargement. However, we did not observe a statistically significant difference, probably due to the small sample size of the study. Further studies with more renal or other visceral PD cases are needed to show systemic microvascular angiopathy in patients with COVID- 19 .

In conclusion, we found that a large proportion of patients with mild-to-moderate COVID-19 had PDs in their lungs and kidneys, which may indicate the presence of systemic microangiopathy with micro-thrombosis. These findings help to understand the physiology of hypoxemia and may have implications regarding the management of patients with COVID-19, such as early indications for thromboprophylaxis or anticoagulation and optimizing strategies for oxygenation.

Funding information The authors state that this work has not received any funding.

\section{Compliance with ethical standards}

Guarantor The scientific guarantor of this publication is Deniz Akata, M.D.

Conflict of interest The authors of this manuscript declare no relationships with any companies whose products or services may be related to the subject matter of the article.

Statistics and biometry No complex statistical methods were necessary for this paper.

Informed consent Written informed consent was not required for this study due to the retrospective design.

Ethical approval Hacettepe University Ethics Board for NonInterventional Studies reviewed and approved the study protocol (Decision no: 17.04.2020 - GO 20/388).
Methodology
- Retrospective
- Observational
- Performed at one institution 


\section{References}

1. Ai T, Yang Z, Hou H et al (2020) Correlation of chest CT and RTPCR testing in coronavirus disease 2019 (COVID-19) in China: a report of 1014 cases. Radiology. https://doi.org/10.1148/radiol. 2020200642

2. Fang Y, Zhang H, Xie J et al (2020) Sensitivity of chest CT for COVID-19: comparison to RT-PCR. Radiology. https://doi.org/10. 1148/radiol.2020200432

3. Xie X, Zhong Z, Zhao W, Zheng C, Wang F, Liu J (2020) Chest CT for typical 2019-nCoV pneumonia: relationship to negative RTPCR testing. Radiology. https://doi.org/10.1148/radiol. 2020200343

4. Jin YH, Cai L, Cheng ZS et al (2020) A rapid advice guideline for the diagnosis and treatment of 2019 novel coronavirus (2019nCoV) infected pneumonia (standard version). Mil Med Res 7:4

5. Salehi S, Abedi A, Balakrishnan S, Gholamrezanezhad A (2020) Coronavirus disease 2019 (COVID-19): a systematic review of imaging findings in 919 patients. AJR Am J Roentgenol. https:// doi.org/10.2214/AJR.20.23034

6. Letko M, Marzi A, Munster V (2020) Functional assessment of cell entry and receptor usage for SARS-CoV-2 and other lineage B betacoronaviruses. Nat Microbiol 5:562-569

7. Sungnak W, Huang N, Bécavin C et al (2020) SARS-CoV-2 entry factors are highly expressed in nasal epithelial cells together with innate immune genes. Nat Med. https://doi.org/10.1038/s41591020-0868-6

8. Varga Z, Flammer AJ, Steiger P et al (2020) Endothelial cell infection and endotheliitis in COVID-19. Lancet. 395:1417-1418

9. Luo W, Yu H, Gou J et al (2020) Clinical pathology of critical patient with novel coronavirus pneumonia (COVID-19). Preprints. 2020020407

10. Tang N, Bai H, Chen X, Gong J, Li D, Sun Z (2020) Anticoagulant treatment is associated with decreased mortality in severe coronavirus disease 2019 patients with coagulopathy. J Thromb Haemost 18:1094-1099

11. Fuld MK, Halaweish AF, Haynes SE, Divekar AA, Guo J, Hoffman EA (2013) Pulmonary perfused blood volume with dual-energy CT as surrogate for pulmonary perfusion assessed with dynamic multidetector CT. Radiology. 267:747-756

12. Aran S, Daftari Besheli L, Karcaaltincaba M, Gupta R, Flores EJ, Abujudeh HH (2014) Applications of dual-energy CT in emergency radiology. AJR Am J Roentgenol 202:314-324

13. Feng Y, Ling Y, Bai T et al (2020) COVID-19 with different severities: a multi-center study of clinical features. Am J Respir Crit Care Med 201:1380-1388

14. Huang G, Gong T, Wang G et al (2020) Timely diagnosis and treatment shortens the time to resolution of coronavirus disease (COVID-19) pneumonia and lowers the highest and last CT scores from sequential chest CT. AJR Am J Roentgenol. https://doi.org/ 10.2214/AJR.20.23078

15. Gervaise A, Bouzad C, Peroux E, Helissey C (2020) Acute pulmonary embolism in non-hospitalized COVID-19 patients referred to CTPA by emergency department. Eur Radiol. https://doi.org/10. 1007/s00330-020-06977-5

16. Grillet F, Behr J, Calame P, Aubry S, Delabrousse E (2020) Acute pulmonary embolism associated with COVID-19 pneumonia detected by pulmonary CT angiography. Radiology. https://doi.org/ 10.1148/radiol.2020201544

17. Lang M, Som A, Mendoza DP et al (2020) Hypoxaemia related to COVID-19: vascular and perfusion abnormalities on dual-energy CT. Lancet Infect Dis. https://doi.org/10.1016/S1473-3099(20) 30367-4

18. Otrakji A, Digumarthy SR, Lo Gullo R, Flores EJ, Shepard JA, Kalra MK (2016) Dual-energy CT: spectrum of thoracic abnormalities. Radiographics. 36:38-52

19. Oudkerk M, Büller HR, Kuijpers D et al (2020) Diagnosis, prevention, and treatment of thromboembolic complications in COVID19: report of the National Institute for Public Health of the Netherlands. Radiology. https://doi.org/10.1148/radiol. 2020201629

20. Carsana L, Sonzogni A, Nasr A et al (2020) Pulmonary postmortem findings in a large series of COVID-19 cases from northern Italy. medRxiv. https://doi.org/10.1101/2020.04.19.20054262

21. Weidman EK, Plodkowski AJ, Halpenny DF et al (2018) Dualenergy CT angiography for detection of pulmonary emboli: incremental benefit of iodine maps. Radiology. 289:546-553

22. Subramaniam S, Scharrer I (2018) Procoagulant activity during viral infections. Front Biosci (Landmark Ed) 23:1060-1081

23. Magro C, Mulvey JJ, Berlin D et al (2020) Complement associated microvascular injury and thrombosis in the pathogenesis of severe COVID-19 infection: a report of five cases. Transl Res. https://doi. org/10.1016/j.trsl.2020.04.007

24. Zhang Y, Xiao M, Zhang S et al (2020) Coagulopathy and antiphospholipid antibodies in patients with Covid-19. N Engl J Med 382:e38

25. Thachil J, Tang N, Gando S et al (2020) ISTH interim guidance on recognition and management of coagulopathy in COVID-19. J Thromb Haemost 18:1023-1026

26. Petri M (2011) Use of hydroxychloroquine to prevent thrombosis in systemic lupus erythematosus and in antiphospholipid antibodypositive patients. Curr Rheumatol Rep 13:77-80

27. Su H, Yang M, Wan C et al (2020) Renal histopathological analysis of 26 postmortem findings of patients with COVID-19 in China. Kidney Int. https://doi.org/10.1016/j.kint.2020.04.003

Publisher's note Springer Nature remains neutral with regard to jurisdictional claims in published maps and institutional affiliations. 